Website: http://revistas.lamolina.edu.pe/index.php/acu/index

(C) Universidad Nacional Agraria La Molina, Lima - Perú

\title{
Plan de marketing para aumentar la cantidad de postulantes del Centro de Admisión y Promoción de la UNALM en el período 2016-2017
}

\author{
Marketing plan to increase the number of applicants to UNALM'S Center of Admission and \\ Promotion in the period 2016-2017
}

\author{
Yulissa Navarro Castillo ${ }^{1}$; Luis Espinoza Villanueva ${ }^{2}$; Christian Encina Zelada ${ }^{3}$
}

\begin{abstract}
Resumen
Con el objetivo de obtener una proyección del aumento en la cantidad de postulantes a la Universidad Nacional Agraria la Molina (UNALM) a través de la implementación de un Plan de Marketing especializado que permita proyectar el aumento progresivo de postulantes al examen de admisión que se realiza cada semestre. Se analizó la base de datos del Centro de Admisión y Promoción (CAP) de la UNALM. En la primera etapa se tabuló e interpretó los datos recolectados en el período del 2010 al 2014, para determinar la situación en la que se encontraba el (CAP), de esa manera generar el enfoque correcto de las estrategias a través de un marketing educativo. En la segunda etapa, se analizaron y filtraron los datos existentes; y se recolectaron nuevos datos para contrarrestar los resultados obtenidos y generar un panorama más amplio de la percepción de los postulantes con respecto al examen de admisión. Se concluye que la mejor estrategia para los fines del Centro de Admisión y Promoción es aumentar la cantidad de postulantes en el examen de admisión y que estos se agruparon en dos clases, las estrategias de diferenciación y las de crecimiento.
\end{abstract}

Palabras clave: Ingreso a Universidad; Proceso de admisión; Plan de marketing; Marketing educativo.

\begin{abstract}
Abstrac
With the aim of obtaining a projection of the increase in the number of applicants to the National Agrarian University la Molina (UNALM) through the implementation of a specialized Marketing Plan that allows projecting the progressive increase of applicants to the admission exam that takes place each semester. The database of the Admission and Promotion Center (CAP) of UNALM was analyzed. In the first stage, the data collected during the period from 2010 to 2014 was tabulated and interpreted, in order to determine the situation in which the CAP was located, in this way generating the correct approach of the strategies through an educational marketing. In the second stage, existing data was analyzed and filtered; and new data were collected to counteract the results obtained and generate a broader picture of the perception of the applicants with respect to the admission exam. It is concluded that the best strategy for the purposes of the Admission and Promotion Center is to increase the number of applicants in the admission exam and that these were grouped into two classes, differentiation strategies and growth strategies.
\end{abstract}

Keywords: University admissions; Selection Process; Marketing Plan; Strategic Marketing.

\section{Introducción}

El problema que identificó el CAP de la UNALM, fue la poca afluencia de postulantes a los exámenes de admisión realizados en los meses de febrero y agosto del año 2010 al 2017; asimismo; se identificó la falta de un plan que oriente las estrategias de marketing dirigido a un público específico en el sector de educación. El CAP cuenta con presupuestos reales designados para la promoción de carreras de la UNALM.

Según Ceballos et al. (2012) para el éxito de un plan de marketing para incrementar el número de estudiantes en un centro educativo se debe utilizar una metodología que consista básicamente en analizar el sector educativo en los diferentes estratos sociales y diseñar estrategias de lanzamiento a parir del análisis elaborado, con un plan de acción y presupuesto para la ejecución de las estrategias planeadas. Asimismo, Salazar (2010) dio a conocer en su plan de marketing el conocimiento de los productos y servicios por parte de los clientes, a causa de un correcto plan de marketing generado por las empresas y organizaciones que desean vender y llegar a los mercados.

En el Marketing Educativo, el proceso de investigación de las necesidades sociales, son para desarrollar servicios educativos para satisfacerlas, acorde a un valor percibido, distribuidas en tiempo y lugar, éticamente promocionadas para generar bienestar entre individuos y organizaciones (Fernández, 2002). Los principales problemas del marketing educativo son: temor al cambio, falta de planificación, no involucrar al personal y no conocer el segmento/producto/servicio. Mientras que los principales objetivos son: identificar las necesidades, la motivación de las familias y de otros colectivos de interés; aumentar su

\footnotetext{
${ }^{1} \mathrm{Mg}$. en Administración Pública por la Universidad de Minho, Braga, Portugal. Email: yulissamnc@gmail.com

${ }^{2}$ Mg. Sc. en Economía Agrícola por la Universidad Nacional Agraria la Molina, Lima, Perú. Email: lespinoza@lamolina.edu.pe

${ }^{3} \mathrm{Mg}$. Sc. en Tecnología de Alimentos por la Universidad Nacional Agraria la Molina, Lima, Perú. Email: cencina@lamolina.edu.pe
} 
satisfacción, planificar la captación del alumnado, mejorar el posicionamiento del centro en el entorno donde trabaja y elaborar acciones comunicativas que reflejen bien la esencia y su propio trabajo (XBD, 2015).

El Plan de marketing tiene una cobertura más limitada que el Plan de negocio, sirve para documentar la forma en que se alcanzarán los objetivos de la organización mediante estrategias y tácticas de marketing específico, partiendo del cliente. También, está vinculado con los planes de otros departamentos dentro de la organización (Kotler y Armstrong, 2012).

Una de las características más útiles e importantes del marketing estratégico consiste en poder planificar, con garantía de éxito, el futuro de la empresa, basándose en las respuestas que se ofrezca a las demandas del mercado, ya que el entorno en el que se posiciona cambia y evoluciona constantemente, el éxito de la empresa dependerá, en gran parte, de la capacidad de adaptación y anticipación a los cambios. Se debe ser capaz de comprender en qué medida y de qué forma los cambios futuros que experimentará el mercado afectarán a la empresa y de establecer las estrategias más adecuadas para aprovecharlas al máximo en beneficio (Muñiz, 2014).

Análisis de mercado, es la recopilación y el análisis de información, en lo que respecta al mundo de la empresa y del mercado, realizados de forma sistemática o expresa, para poder tomar decisiones dentro del campo del marketing estratégico y operativo. Se trata, en definitiva, de una potente herramienta, que debe permitir a la empresa obtener la información necesaria para establecer las diferentes políticas, objetivos, planes y estrategias más adecuadas a sus intereses (Muñiz, 2014).

El entorno, en el caso del marketing, además de influir en la estrategia, puede influir en las acciones que realizan la fuerza de ventas y las acciones de comunicación para satisfacer sus mercados. Desde una perspectiva del marketing, el estudio del entorno es crítico porque cualquier cambio en las variables del medio ambiente repercute tanto en la empresa como en el mercado. Como consecuencia, la noción de intercambio y transacción entre los componentes del concepto de mercado puede verse afectado. Por la conformación de variables, con diverso tipo de influencia sobre la empresa, se le divide en tres clases: macroentorno, entorno operativo y entorno interno (Rivera y López, 2007).

El Macroentorno, está formado por las variables que están más lejos del control de la empresa y su influencia no es directa. Aunque dichas variables no pueden ser controladas por la empresa, deben ser tomadas en cuenta porque pueden condicionar la viabilidad de las acciones competitivas. Este tipo de entorno está compuesto por las condiciones internacionales de tipo social, político, económico y tecnológico (Rivera y López, 2007).

El entorno operativo agrupa a las variables que están relacionadas con el funcionamiento competitivo de la empresa. En este entorno están incluidas las variables que pueden afectar a las operaciones de la empresa por satisfacer a sus mercados. Son las variables sobre las que se tiene mediano control y que ejercen una influencia directa y a corto plazo: los proveedores, los competidores, los distribuidores y los clientes finales (Rivera y López, 2007). Mientras que el entorno interno es aquel que influye a corto plazo sobre la empresa y sobre el cual esta tiene más influencia. En este tipo de entorno se incluyen las diversas áreas funcionales de la empresa, así como los diversos niveles jerárquicos que forman la estructura organizativa. También, se incluyen las acciones que desarrolla la empresa para satisfacer a sus mercado (Rivera y López, 2007).

El marketing mix es un análisis de estrategia de aspectos internos, desarrollada comúnmente por las empresas para analizar cuatros variables básicas de su actividad: producto, precio, distribución y promoción (Kotler y Armstrong, 2012).

Un producto es cualquier cosa que se puede ofrecer en un mercado para su atención, adquisición, uso o consumo y que podría satisfacer un deseo o una necesidad. Sin embargo, el concepto de producto no está limitado a objetos físicos; cualquier cosa que pueda satisfacer una necesidad se puede llamar producto. Además de los bienes tangibles, los productos incluyen servicios, que son actividades o beneficios que se ofrecen a la venta y que son básicamente intangibles y no tienen como resultado la propiedad de algo (Kotler y Armstrong, 2012).

La distribución es una herramienta de la mercadotecnia que incluye un conjunto de estrategias, procesos y actividades necesarios para llevar los productos desde el punto de fabricación hasta el lugar en el que esté disponible para el cliente final (consumidor o usuario industrial) en las cantidades precisas, en condiciones óptimas de consumo uso y en el momento y lugar en el que los clientes lo necesitan y/o desean (Thompson, 2007).

El precio, es la cantidad de dinero que se cobra por un producto o servicio. En términos más amplios, el precio es la suma de los valores que los consumidores dan a cambio de los beneficios de tener o usar el producto o servicio (Kotler y Armstrong, 2012).

La promoción, es la cuarta herramienta del marketingmix, incluye las distintas actividades que desarrollan las empresas para comunicar los méritos de sus productos y persuadir a su público objetivo para que compren (Kotler et al., 2004).

La UNALM, a través del CAP, requiere de un correcto plan de marketing, a fin de establecer un posicionamiento adecuado en el mercado educacional del país. Por tal razón, el objetivo del presente estudio fue establecer un plan de marketing educativo acorde con las necesidades del CAP, para lograr el aumento de postulantes a la UNALM.

\section{Materiales y métodos}

El presente estudio se realizó en la UNALM, ubicada en el distrito de La Molina, Lima, Perú. Se utilizaron las instalaciones del CAP-UNALM para la obtención y procesamiento de los datos utilizados para la investigación. 
El tipo de investigación fue descriptivo. El estudio se realizó en el rango longitudinal ya que se analizaron las variables a lo largo de cinco años, se utilizaron las dos áreas: retrospectiva, que analizó a los postulantes desde los últimos cinco años y prospectiva, que analizó y proyectó en base a datos históricos los objetivos del estudio. La técnica utilizada para la realización de la investigación fue el análisis directo de la base de datos proporcionada en el CAP, además de encuestas vía electrónica y grupos focales.

Población y muestra, estuvo conformada por los postulantes de los últimos cinco años, de los dos procesos de admisión, lo que convierte el estudio en un censo poblacional de postulantes al examen de Admisión de la Universidad Agraria la Molina que se realizan de manera semestral dando un total de diez procesos los cuales fueron divididos por proceso en: verano e invierno; dando una población total de 28331 postulantes, motivo por el cual no se ha determinado una muestra.

La información fue obtenida de la base de dato y de las encuestas realizadas por el CAP de la UNALM, récord de postulantes de los diferentes procesos de admisión y las técnicas de marketing utilizadas en cada una de ellas, información sobre la estrategia de marketing utilizado por la competencia más cercana a la UNALM. Documentos de la organización como el Manual de Organización y funciones, planes de marketing diseñados anteriormente y documentos de presupuesto.

Se utilizó un computador, materiales de oficina hojas bond, copias, lapiceros, teléfono, transporte, software para análisis de datos (Excel y SPSS $® 33)$.

\section{Resultados y discusión}

Los resultados obtenidos de la base de datos del CAP $\mathrm{y}$ del formulario que llenaron los postulantes en cada proceso de admisión, brindaron una visión más clara para la orientación del plan de marketing y la solución a los objetivos de la investigación.

Para la proyección del aumento de postulantes a la universidad para la investigación se utilizó un rango de aumento de postulantes del $5 \%$ al $10 \%$, en el margen de proyección conservadora y optimista respectivamente, en la Figura 1, proyección al 5\% del aumento de postulantes, se muestra el resultado proyectado para los postulantes en el escenario conservador del $5 \%$ de aumento con la implementación del plan de marketing.

Como se puede observar la cantidad de postulantes en el primer examen de admisión se proyecta a más de tres mil postulantes, cifra que ha sido alcanzada por primera vez en el año 2011(Centro de Admisión y promoción, 2014) en el ciclo verano, mientras en el ciclo invierno la cifra significativa se proyecta al año 2016.

Para la segunda proyección se utilizó el escenario optimista con el $10 \%$ de aumento de postulantes se gráfica en la Figura 2, proyección al 10\% del aumento de postulantes.

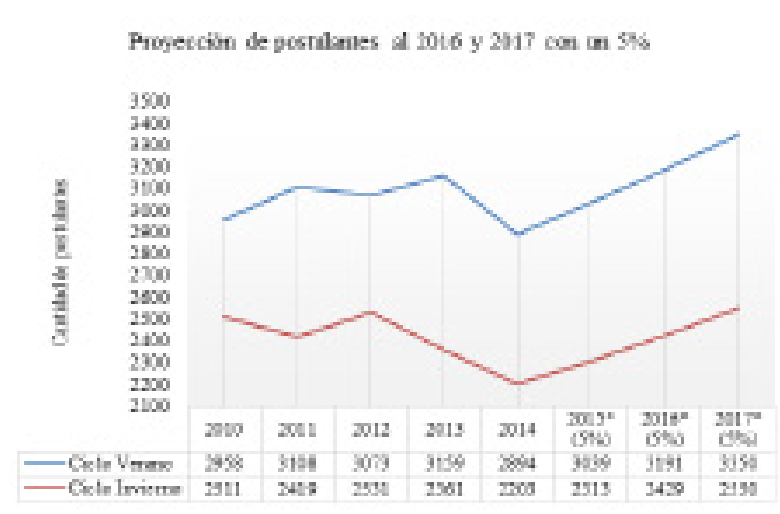

Figura 1. Proyección al 5\% del aumento de postulantes (*) Datos proyectados al 2016 y 2017.

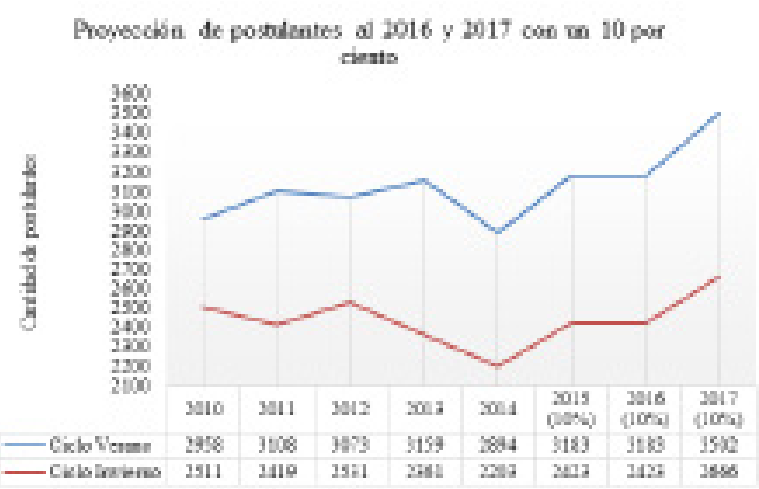

Figura 2. Proyección al 10\% del aumento de postulantes (*) Datos proyectados al 2016 y 2017.

El escenario optimista sobrepasa el record alcanzado por el CAP durante todos sus años, en el año 2016 con poco más de 3500 postulantes proyectados, en paralelo el ciclo invierno proyecta una cifra considerable en el año 2016, cifra mayor a las obtenidas dentro de los años de estudio.

El público objetivo fue definido en base a la información colectada por el CAP de la UNALM analizando las variables: Grupo de Etario, Tipo de Colegio y Provincia de procedencia, las edades promedio en el examen de admisión tienen un rango con frecuencias de más del 90\% los postulantes que se encuentran en el rango de edad desde los 16 años hasta los 23 años.

En la Tabla 1, porcentaje de grupos etarios: período 2010 - 2014, se han separado las edades por grupos etarios según su participación en el examen de admisión los grupos son: menores de 15 años, de 16 a 23 años, de 24 a 30 años y mayores de 30 años.

Región de procedencia, los datos de la procedencia de los postulantes se analizaron agrupándolos en cada una de las regiones del Perú y además se considera un grupo adicional que es: extranjero Figura 3, porcentaje promedio de procedencia de postulantes 2010 - 2014, se aprecia la participación obtenida con la agrupación de los grupos con un porcentaje menor a 4\%. Para el estudio se determinó 
que el enfoque geográfico del plan de marketing sería en la Región Lima que presentó el 80,48\% de participación en promedio dentro de los cinco años de estudio.

Tabla1. Porcentaje de grupos etarios: período

\begin{tabular}{cccccc}
\multicolumn{7}{c}{$2010-2014$} \\
\hline $\begin{array}{c}<15 \\
\text { años }\end{array}$ & $\begin{array}{c}16-23 \\
\text { años }\end{array}$ & $\begin{array}{c}24-30 \\
\text { años }\end{array}$ & $\begin{array}{c}>30 \\
\text { años }\end{array}$ & Total \\
\hline $\begin{array}{c}\text { Promedio ciclo } \\
\text { verano }\end{array}$ & 23 & 3029 & 87 & 13 & 3153 \\
$\begin{array}{c}\text { Porcentaje } \\
\text { Pornedio ciclo }\end{array}$ & $0,74 \%$ & $96,07 \%$ & $2,77 \%$ & $0,43 \%$ & $100 \%$ \\
\hline $\begin{array}{c}\text { Promedio } \\
\text { invierno }\end{array}$ & 7 & 2422 & 75 & 10 & 2513 \\
Porcentaje & $0,28 \%$ & $96,36 \%$ & $2,98 \%$ & $0,38 \%$ & $100 \%$ \\
\hline
\end{tabular}

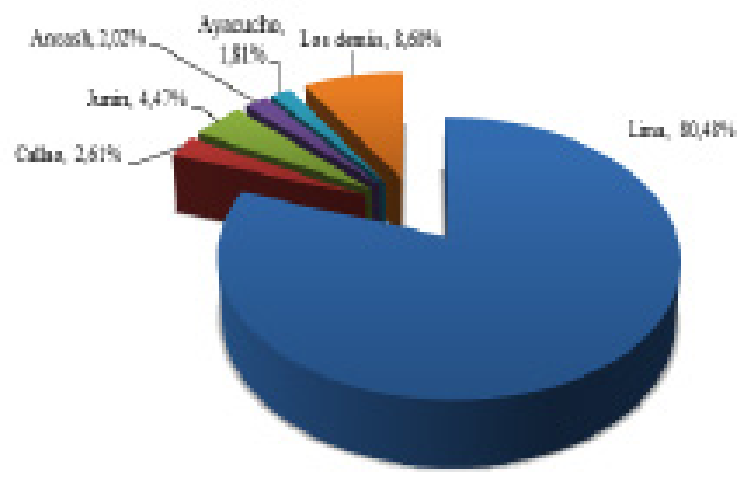

Figura 3. Porcentaje de procedencia de postulantes 2010 $-2014$

Según la LEY No 27658 en el Artículo 4. De la Ley Marco de Modernización de Gestión del Estado afirma que: El proceso de modernización de la gestión del Estado tiene como finalidad fundamental la obtención de mayores niveles de eficiencia del aparato estatal, de manera que se logre una mejor atención a la ciudadanía, priorizando y optimizando el uso de los recursos públicos. El objetivo es alcanzar un Estado: al servicio de la ciudadanía, con canales efectivos de participación ciudadana, descentralizado y desconcentrado, transparente en su gestión, con servidores públicos calificados y adecuadamente remunerados; y fiscalmente equilibrado.

El tipo de colegio, como se pudo observar en los resultados obtenidos de la base de datos del CAP, en el año 2011 hasta el 2014 se ha visto incrementado la participación de colegios privados en los exámenes de admisión del examen de admisión, motivo por el cual el plan de marketing tuvo un enfoque mayor en aquellos colegios privados del sector B y C; así también como los colegios estatales con poblaciones de más de 1000 alumnos priorizando los colegios modelo del estado.

La participación promedio de los colegios del tipo público y privado se ve diferenciada con favor a los colegios privados lo cual concuerda con el análisis te comportamiento que se hizo líneas arriba. En la Figura 4, porcentaje de participación promedio por tipo de colegio verano y la Figura 5, porcentaje de participación promedio por tipo de colegio - invierno se aprecia de manera gráfica.

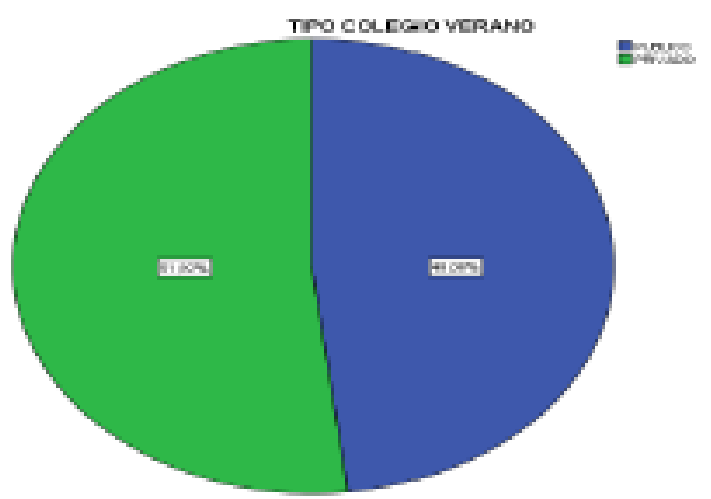

Figura 4. Porcentaje de participación promedio por tipo de colegio - verano

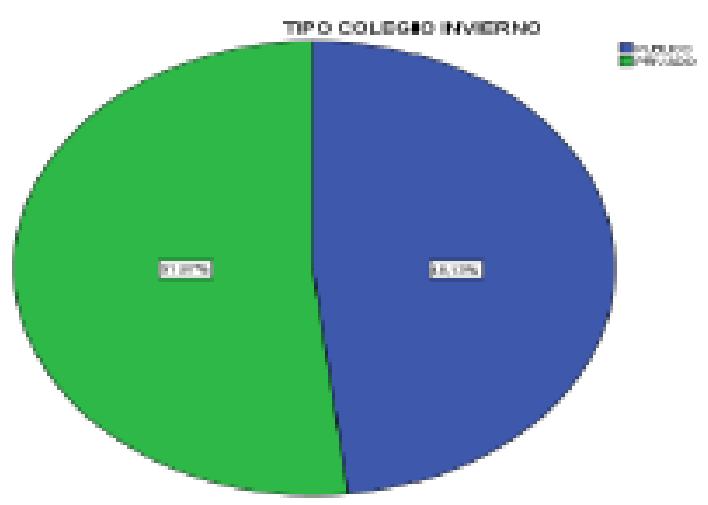

Figura 5. Porcentaje de participación promedio por tipo de colegio - invierno

Carreras de menor porcentaje. Durante el estudio se encontró las carreras con menor porcentaje en los diferentes exámenes de admisión siendo en los 10 procesos observados, las mismas carreras profesionales aquellas que ocupan los últimos lugares de preferencia entre los postulantes; en la Tabla 2, valores estadísticos de las carreras elegidas como primera opción promedio de verano e invierno, se encuentra la desviación estándar y el promedio encontrado al analizar de manera estadística el promedio aritmético de las opciones elegidas por los postulantes en el período de estudio.

En la Tabla 3, la frecuencia del promedio de la primera opción elegida por los postulantes en los ciclos de verano, se presenta las frecuencias estadísticas de los promedios que se obtuvieron en el período de estudio así mismo se puede observar en la Tabla 4, frecuencia del promedio de la primera opción elegida por los postulantes en los ciclos de invierno, los valores obtenidos con los postulantes en los ciclos de mitad de año.

En la Figura 6, el porcentaje de postulantes al examen de admisión período 2010 - 2014 en su primera opción - ciclo verano, se puede observar las preferencias de los postulantes en el ciclo verano analizado con un promedio 
simple que posteriormente se analizaron mediante el paquete estadístico SPSS $®$ para corroborar los datos obtenidos.

Tabla 2. Valores estadísticos de las carreras elegidas como primera opción promedio de verano e invierno

\begin{tabular}{lccc}
\hline \multicolumn{3}{c}{ Estadísticos } \\
\hline & & Opción & Opción \\
& & Verano & Invierno \\
\hline $\mathrm{N}$ & Válido & 3038 & 2405 \\
& Perdidos & 0 & 633 \\
Moda & 22 & 22 \\
Desviación estándar & 22,894 & 23,114 \\
\multicolumn{2}{l}{ Varianza } & 524,141 & 534,249 \\
\hline
\end{tabular}

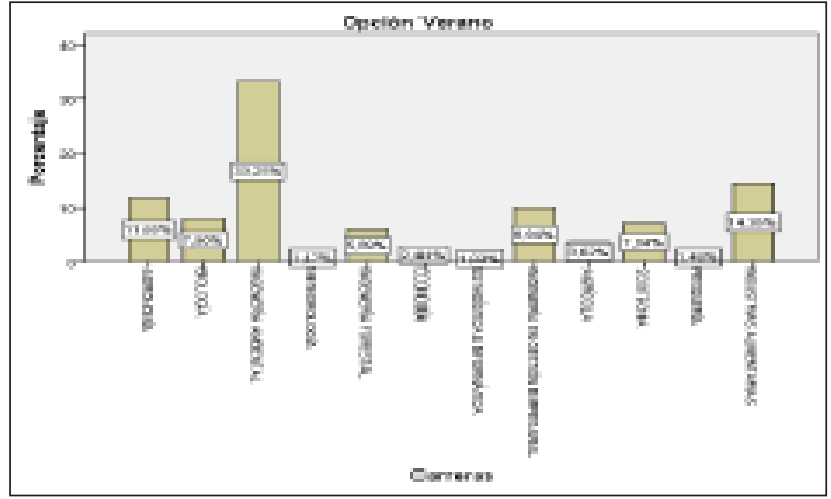

Figura 6. Porcentaje de Postulantes al examen de admisión período 2010 - 2014 en su primera opción - Ciclo Verano

Tabla 3. Frecuencia del promedio de la primera opción elegida por los postulantes en los ciclos de verano

\begin{tabular}{lcccc}
\hline \multicolumn{5}{c}{ Opción Verano } \\
& Frecuencia & $\%$ & \% válido & \% acumulado \\
\hline Agronomía & 354 & 11,7 & 11,7 & 11,7 \\
Biología & 240 & 7,9 & 7,9 & 19,6 \\
Ingeniería ambiental & 1010 & 33,2 & 33,2 & 52,8 \\
Meteorología & 43 & 1,4 & 1,4 & 54,2 \\
Ingeniería forestal & 179 & 5,9 & 5,9 & 60,1 \\
Economía & 74 & 2,4 & 2,4 & 62,5 \\
Válido Estadística e informática & 31 & 1,0 & 1,0 & 63,6 \\
Ingeniería en gestión empresarial & 302 & 9,9 & 9,9 & 73,5 \\
Agrícola & 110 & 3,6 & 3,6 & 77,1 \\
Zootecnia & 214 & 7,0 & 7,0 & 84,2 \\
Pesquería & 45 & 1,5 & 1,5 & 85,6 \\
Industrias alimentarias & 436 & 14,4 & 14,4 & 100,0 \\
\hline Total & 3038 & 100,0 & 100,0 & \\
\hline
\end{tabular}

Tabla 4. Frecuencia del promedio de la primera opción elegida por los postulantes en los ciclos de

\begin{tabular}{lcccc}
\multicolumn{5}{c}{ invierno } \\
\cline { 2 - 5 } Opción Invierno & & \\
\cline { 2 - 5 } & Frecuencia & $\%$ & \% válido & \% acumulado \\
\hline Agronomía & 306 & 10,1 & 12,7 & 12,7 \\
Biología & 195 & 6,4 & 8,1 & 20,8 \\
Ingeniería ambiental & 728 & 24,0 & 30,3 & 51,1 \\
Meteorología & 33 & 1,1 & 1,4 & 52,5 \\
Ingeniería forestal & 167 & 5,5 & 6,9 & 59,4 \\
Economía & 57 & 1,9 & 2,4 & 61,8 \\
Válido & 25 & 0,8 & 1,0 & 62,8 \\
Estadística e informática & 246 & 8,1 & 10,2 & 73,1 \\
Ingeniería en gestión empresarial & 91 & 3,0 & 3,8 & 76,8 \\
Agrícola & 164 & 5,4 & 6,8 & 83,7 \\
Zootecnia & 38 & 1,3 & 1,6 & 85,2 \\
Pesquería & 355 & 11,7 & 14,8 & 100,0 \\
Industrias alimentarias & 2405 & 79,2 & 100,0 & \\
Total & 633 & 20,8 & & \\
Perdidos Sistema & 3038 & 100,0 & & \\
\hline \multicolumn{1}{c}{ Total } &
\end{tabular}


Los resultados obtenidos de los último cinco exámenes de admisión, del 2010 al 2014, ciclo verano, las carreras que presentaron menor acogida fueron: Estadística e Informática $(1,03 \%)$, Meteorología $(1,42 \%)$ y Pesquería $(1,48 \%)$; las cuales tuvieron una acogida menor al dos por ciento. Por otro lado, las preferencias se mantuvieron los últimos cinco años donde más de $33 \%$ de los postulantes optaron por la carrera de Ingeniería Ambiental, seguida de Industrias Alimentarias con más del 14\% y Agronomía con un porcentaje mayor a $11 \%$. Del mismo modo se realizó la evaluación para el ciclo invierno obteniendo los resultados que se muestran en la Figura 7. Similar resultado se obtuvo al ciclo verano en los resultados de los últimos cinco exámenes de admisión tomados en el período de 2010 al 2014 en el ciclo invierno. De la misma manera, las preferencias se mantuvieron al pasar del tiempo más de $30 \%$ de los postulantes optaron por la carrera de Ingeniería Ambiental; la cual disminuyó en un 3\% en relación a los resultados del ciclo verano, seguida con más del 14\% la carrera de Industrias Alimentarias la cual mantuvo su porcentaje y en tercer lugar se situó una vez más la carrera de Agronomía con un porcentaje mayor a 12; resultado mayor en $1 \%$ al ciclo de verano.

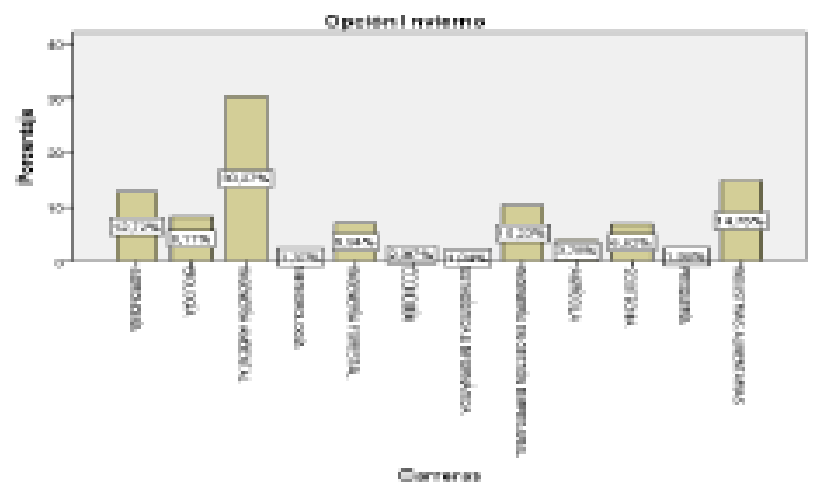

Figura 7. Porcentaje de postulantes al examen de admisión período 2010 - 2014 en su primera opción de ciclo invierno

\section{Conclusiones}

La variación promedio del ciclo verano e invierno fue de $-0,41 \%$ y $-3,11 \%$ respectivamente, durante el período de estudio (2010 - 2014). El público objetivo del examen de admisión de pregrado son aquellos alumnos que hayan concluido el quinto de secundaria, se encuentren en academias o grupos de estudios entre 16 y 23 años del sector socioeconómico $\mathrm{C}$ y $\mathrm{D}$. Las carreras que presentaron menor acogida en los últimos cinco años fueron las carreras de: Estadística e Informática $(1,04 \%)$, Meteorología $(1,39 \%)$ y la carrera de Pesquería $(1,53 \%)$; las cuales no pasaron el dos por ciento cada una en el período evaluado. En base al análisis FODA realizado se determinaron que las mejores estrategias para los fines del Centro de Admisión y Promoción (Aumentar la cantidad de postulantes en el examen de admisión) que se agruparon en dos clases, las estrategias de Diferenciación y las de Crecimiento. El costo de la implementación del Plan de Marketing asciende a un monto de S/. 1'145700,00 en los cuatro procesos de admisión proyectados al 2016 y 2017 siendo un $20 \%$ del ingreso bruto de los exámenes que se proyectaron.

\section{Literatura citada}

Centro de Admisión y Promoción - UNALM. 2014. Base de datos - 2014.

Ceballos, A.M.; Arévalo, B. y Giraldo, J. 2012. Plan de Marketing para incrementar el número de estudiantes en el Centro Educativo la Sabiduría de la Ciudad de Barranquilla. Escenarios 10 (1): 29-39pp.

Fernández, R. 2002. Fundamentos de Mercadotecnia. Editorial Thompson. México. 11-12pp.

Kotler, P. y Armstrong, G. 2012. Marketing. 14º edición. Pearson. México. 5; 7; 136pp.

Kotler, P.; Amstrong, G.; Cámara, D. y Cruz, I. 2004. Marketing. $10^{\circ}$ edición, Pearson, España, 75pp.

LEY N ${ }^{\circ}$ 27658. Ley Marco de Modernización de la Gestión del Estado. Disponible en: http://www.minagri.gob.pe/ portal/download/pdf/cetsar/ley-modernizacion.pdf

Muñiz, R. 2014. Marketing en el Siglo XXI. Disponible en: (http://www.marketing-xxi.com/Marketing-sigloxxi. html).

Rivera, J. y López, M. 2007. Dirección de marketing fundamento y aplicaciones. $1^{\circ}$ edición. ESIC Editorial. España. 52, 53pp.

Salazar, R. 2010. Plan estratégico de marketing para el Instituto Tecnológico Superior Aeronáutico de la Fuerza Aérea Ecuatoriana. Instituto Tecnológico Superior Aeronáutico de la Fuerza Aérea Ecuatoriana. Ecuador. Disponible en: http://repositorio.espe.edu.ec/ handle/21000/1096.

Thompson, I. 2007. Artículo "Definición de distribución”. Publicado en abril del 2007.

XBD, Grupo XABIDE. 2015. Y de repente... el Marketing educativo. Disponible en: (http://www.grupoxabide. com/y-de-repente-el-marketing-educativo/) 